\title{
The QT Interval in Research
}

\author{
John Somberga, b
}

In this issue of the Journal Al-Zakhaui authors a paper "Corrected QT Interval Prolongation, Elevated Troponin and Mortality Among Hospitalized COVID-19 Patients", suggesting another area that a QT interval measurement may be useful [1]. Having an inexpensive, easy way to obtain test that can help predict risk for an adverse outcome in coronavirus disease 2019 (COVID-19) patients would be useful. This is especially the case as we have an expanded therapeutic armamentarium to treat more severe COVID disease.

QT measurements have been used for a long time to predict arrhythmic risk. Repolarization abnormalities may be a sensitive marker for myocardial injury. Having just finished editing a book "On the QT" (www.amazon.com, ISBN: 978-17368527-0-5), the QT interval is a complex subject. It indeed is very useful in assessing arrhythmic risk from drugs, both antiarrhythmic agents, as well as non-cardiac drugs. For the anti-arrhythmic drug amiodarone, it may well predict anti-arrhythmic efficacy. Measuring the QT interval can be difficult. One possible limitation in the study published in this issue of the Journal was the use of a standard electrocardiogram (ECG) with automatic machine generated readings. Often these readings can be problematic, given the difficulty in determining the end of the QT interval. Rate correction formulas may also be problematic at fast as well as very slow heart rates. But especially the circadian nature of QT duration distribution can confound studies with QT measurements made at different times of the day.

Additional information can be obtained by measuring the QT dispersion (QTd) by calculating the longest less the shorter QT interval on the 12-lead ECG. There has been considerable debate as to the utility of QTd. However, many studies have correlated QTd with outcome in myocardial infarction (MI) patients, ischemia, myocardial hypertrophy, cardiomyopathy, amyloid heart disease, to list but a few of the areas evaluated. While many studies found a correlation between QTd and outcome, others have failed to do so perhaps because of the difficulty in measuring QT offset and circadian variation, which causes a high variability in QT measurements that obscure the physiologic information provided. The numerical values in QT and QTd changes are small, and the errors introduced into the measurements from variability due to QT offset issues and circadian changes magnify errors.
The other concern I have with studies involving the QT/QTd is that on the basis of a single measurement in a patient, a lot of decisions can be made and hypothesis generated. It would be far better to obtain multiple QT measurements, best of all 24-h QT recordings to assess the state of repolarization in a given patient.

Careful and multiple QT measurement, as well as taking into account the time of the day the QT is determined may improve QT and QTd readings, making this simple evaluation clinically useful as well as the basis for consequential cardiovascular research.

\section{Acknowledgments}

None to declare.

\section{Financial Disclosure}

None to declare.

\section{Conflict of Interest}

None to declare.

\section{Data Availability}

The author declares that data supporting the findings of this study are available within the article.

\section{Reference}

1. Al-Zakhari R, Atere M, Lim W, Abdulrahman M, Akhtar S, Sheets N, Joyce T, et al. Corrected QT interval prolongation, elevated troponin, and mortality in hospitalized COVID-19 patients. Cardiol Res. 2021;12(4):258-264.

Manuscript submitted May 12, 2021, accepted May 17, 2021

Published online July 9, 2021

aCardiology \& Pharmacology, Rush University, Chicago, IL 60612, USA.

Email: John_Somberg@rush.edu

${ }^{b}$ Editor-in-Chief, Cardiology Research

doi: https://doi.org/10.14740/cr1280 